\title{
Efeito do cloreto de sódio sobre as respostas fisiológicas e controle de helmintos monogenóides em tambaqui (Colossoma macropomum)
}

\author{
Edsandra Campos CHAGAS ${ }^{*}$, Lucelle Dantas de ARAÚJO², Levy de Carvalho GOMES ${ }^{3}$, José Celso de \\ Oliveira MALTA ${ }^{4}$, Angela Maria Bezerra VARELLA ${ }^{4}$
}

\begin{abstract}
RESUMO
O objetivo deste trabalho foi avaliar o efeito do sal $(\mathrm{NaCl})$ sobre as respostas fisiológicas e controle de helmintos monogenóides em tambaqui (Colossoma macropomum). Para isso, juvenis de tambaqui (42,38 $\pm 0,47 \mathrm{~g} ; 13,90 \pm 0,06 \mathrm{~cm})$ foram transferidos para tanques de $350 \mathrm{~L}$ e aclimatados por um período de 24 horas. Os ensaios foram conduzidos utilizando as concentraçôes de 0, 2, 4, 6 e $8 \mathrm{~g}$ de sal comum $\mathrm{L}^{-1}$ de água, nos tempos de exposição de 30, 60 e 120 minutos, com três repetiçóes por tratamento. A tolerância dos tambaquis ao sal e a eficácia deste sobre os helmintos monogenóides foi avaliada mediante análise dos indicadores fisiológicos (glicose, cloretos, sódio e potássio plasmático) e parasitológicos (número total de parasitas e prevalência). Tambaquis expostos a $4 \mathrm{~g} \mathrm{NaCl} \mathrm{L}^{-1}$ por 120 minutos, 6 e $8 \mathrm{~g} \mathrm{NaCl} \mathrm{L}^{-1}$ por 60 e 120 minutos apresentaram maior elevação da glicose plasmática e na concentração de $8 \mathrm{~g} \mathrm{NaCl} \mathrm{L}^{-1}$ por 120 minutos maiores níveis de cloreto plasmático. A prevalência de monogenóides nas brânquias foi de $100 \%$ e todas as concentraçóes de sal avaliadas neste estudo não foram eficazes na redução dos helmintos monogenóides em tambaqui.
\end{abstract}

PALAVRAS-CHAVE: estresse, fisiologia, sanidade, monogenóides, piscicultura.

\section{Effect of sodium chloride on physiological responses and monogenean control in tambaqui (Colossoma macropomum)}

\section{ABSTRACT}

The objective of this work was to evaluate the effect of salt $(\mathrm{NaCl})$ on physiological responses and monogenean control in tambaqui (Colossoma macropomum). Juveniles of tambaqui $(42.38 \pm 0.47 \mathrm{~g} ; 13.90 \pm 0.06 \mathrm{~cm}$ ) were transferred to $350 \mathrm{~L}$ tanks and acclimated for a period of 24 hours. The assays were conducted using the concentrations of $0,2,4,6$ and $8 \mathrm{~g}$ of $\mathrm{NaCl}$ $\mathrm{L}^{-1}$ of water, exposed to 30,60 and 120 minutes, with three replicates in each treatment. The tambaqui tolerance to salt and the efficacy against monogenean were evaluated by physiological analysis (glucose, chloride, sodium and potassium plasmatic concentrations) and parasitic indices (total number of parasites and prevalence). Tambaquis exposed to $4 \mathrm{~g} \mathrm{NaCl} \mathrm{L}^{-1}$ for 120 minutes, 6 and $8 \mathrm{~g} \mathrm{NaCl} \mathrm{L}^{-1}$ for 60 and 120 minutes showed increased glucose levels while those exposed to $8 \mathrm{~g} \mathrm{NaCl} \mathrm{L}^{-1}$ for 120 minutes presented increased chloride levels. The prevalence of monogeneans in the gills of tambaqui was $100 \%$ and the salt concentrations evaluated in this study were not effective in the reduction of monogenean parasites.

KEYWORDS: stress, physiology, fish health, monogeneans, fish culture.

\footnotetext{
1 Embrapa Amazônia Ocidental, Manaus, AM, Brasil. E-mail: edsandra.chagas@cpaa.embrapa.br

2 Programa de capacitação, Embrapa Amazônia Ocidental, Manaus, AM, Brasil.

${ }^{3}$ Centro Universitário Vila Velha, Vila Velha, ES, Brasil. E-mail: levy.gomes@uvv.br

${ }^{4}$ Instituto Nacional de Pesquisas da Amazônia, Manaus, AM, Brasil. E-mail: jcmalta@inpa.gov.br; avarella@inpa.gov.br
} 


\section{INTRODUÇÃO}

Nos últimos anos a criação de espécies nativas vem crescendo e no caso do tambaqui (Colossoma macropomum), sua criação tem se expandido nas regióes Norte, Nordeste, Centro-oeste e Sudeste (IBAMA 2007), atingindo $46 \mathrm{mil}$ toneladas em 2009, o que representa $14 \%$ do total de pescado proveniente da piscicultura continental (BRASIL 2010). A expansão na criação dessa espécie é atribuída ao seu excelente potencial para produção intensiva, principalmente pela fácil obtenção de juvenis, bom potencial de crescimento, alta produtividade, resistência a baixos níveis de oxigênio dissolvido e excelente utilização de alimentos (Saint-Paul 1986; Val et al. 1998; Melo et al. 2001; Araújo-Lima e Gomes 2005), sendo a espécie mais criada na região Norte do Brasil (IBAMA 2005), alcançando $3 \mathrm{~kg}$ de peso em 12 meses de criação em sistemas de viveiros e barragens (Melo et al. 2001; Araújo-Lima e Gomes 2005). Entretanto, um dos principais problemas relacionados à criação do tambaqui é a ocorrência de doenças parasitárias e bacterianas (Malta et al. 2001; Silva 2001; Varella et al. 2003; Araújo-Lima e Gomes 2005).

$\mathrm{Na}$ criação intensiva do tambaqui há o registro de ocorrência de vários parasitos com potencial patogênico. Entre eles encontram-se os mixosporídeos, os crustáceos, os acantocéfalos, e ainda os helmintos monogenóides (Malta et al. 2001; Araújo et al. 2009; Maciel et al. 2011), sendo que este último grupo são os que apresentam altos valores de intensidade parasitária registrados em peixes cultivados (Varella et al. 2003). Com relação aos monogenóides, as espécies de maior ocorrência são Anacanthorus spathulatus, Linguadactyloides brinkmanni e Notozothecium sp. (Malta et al. 2001; Varella et al. 2003; Thatcher 2006). Altas taxas de infestaçóes parasitárias podem afetar o desempenho dos peixes sob cultivo e aumentar a taxa de mortalidade (Pavanelli et al. 2008).

Os monogenóides caracterizam-se, principalmente, pela presença de um aparelho de fixação localizado geralmente na parte posterior do corpo, o haptor. Esta estrutura é formada por uma série de ganchos, barras e âncoras, que são introduzidos principalmente nas brânquias dos peixes para fixação. Provoca uma série de reaçóes, podendo culminar em uma hipersecreção de muco, o que poderia levar os animais à morte por asfixia, ou ainda provocar lesóes facilitando a penetração de agentes secundários, como fungos e bactérias (Thatcher e Brites Neto 1994), representando um dos fatores limitantes para produção de peixes, tornando necessária medidas alternativas para o seu controle.

A utilização de medicamentos na piscicultura pode apresentar riscos à segurança alimentar dos consumidores de peixes, bem como proporcionar lesôes tóxicas aos peixes expostos a estes fármacos e efeitos deletérios sobre o ecossistema aquático (Tavechio et al. 2009). Dessa forma, a utilização de produtos alternativos, como o sal comum $(\mathrm{NaCl})$, muito empregado em banhos terapêuticos para o controle de monogenóides em peixes (Kabata 1985; Pavanelli et al. 2008), pode reduzir drasticamente a utilização de quimioterápicos e antimicrobianos na criação do tambaqui, proporcionando melhores condiçóes de higidez para estes, maior biosseguridade e sustentabilidade ao sistema de produção, bem como segurança alimentar para os consumidores. Portanto, o objetivo deste trabalho foi avaliar o efeito do sal nas respostas fisiológicas e no controle de helmintos monogenóides em tambaqui (Colossoma macropomum).

\section{MATERIAL E MÉTODOS}

Juvenis de tambaqui, com peso médio de 42,38 $\pm 0,47$ g e comprimento total médio de $13,90 \pm 0,06 \mathrm{~cm}$ foram aclimatados em viveiro de $200 \mathrm{~m}^{2}$ sem renovaçáo de água e aeraçáo, na densidade de 1 peixe $\mathrm{m}^{2}$, durante 30 dias, onde receberam ração comercial extrusada para peixes onívoros com $28 \%$ de proteína bruta (PB). Após esse período, os peixes foram distribuídos em tanques de $350 \mathrm{~L}$, os quais eram dotados de sistemas estáticos, com aeração constante. Em cada tanque experimental $(\mathrm{n}=15)$ foram estocados 15 peixes, perfazendo cinco tratamentos com três repetiçóes, num delineamento inteiramente casualizado. Os peixes foram aclimatados nas unidades experimentais 48 horas antes do início dos banhos terapêuticos.

$\mathrm{Na}$ realização dos ensaios foram avaliadas cinco concentraçóes de sal: 0, 2, 4, 6 e $8 \mathrm{~g} \mathrm{NaCl} \mathrm{L}^{-1}$, em exposiçóes de 30, 60 e 120 minutos. Nesse período, os parâmetros de qualidade de água das unidades experimentais foram monitorados durante a execução dos ensaios. Os valores de $\mathrm{pH}(6,65 \pm 0,01)$ foram obtidos com auxílio de um $\mathrm{pHmetro}$ digital ( $\mathrm{pH} 100$, YSI Environmental); a temperatura da água $\left(26,96 \pm 0,28{ }^{\circ} \mathrm{C}\right)$, o oxigênio dissolvido $(6,71 \pm 0,02 \mathrm{mg}$ $\left.\mathrm{L}^{-1}\right)$ e as salinidades $(0,03 \pm 0,03 ; 2,03 \pm 0,03 ; 3,93 \pm 0,03$; $\left.5,93 \pm 0,03 ; 8,00 \pm 0,06 \mathrm{~g} \mathrm{~L}^{-1}\right)$ foram obtidas utilizando um monitor YSI 85.

Antes do período experimental, foi realizada a análise parasitológica para quantificação dos monogenóides nas brânquias de tambaqui, utilizando uma amostra de 25\% dos peixes estocados no viveiro. Após a realização dos banhos terapêuticos, nos intervalos de 30, 60 e 120 minutos, foram coletados quinze peixes de cada tratamento (cinco por repetição), os quais foram destinados à avaliação fisiológica e parasitológica. Deste total, apenas nove peixes de cada tratamento (três por repetição) foram utilizados na coleta sanguínea, a qual foi realizada com os peixes previamente anestesiados com $100 \mathrm{mg} \mathrm{L}^{-1}$ de benzocaína (Gomes et al. 2001), sendo submetidos à venopunção caudal (1 mL) utilizando seringas estéreis com volume de $3 \mathrm{~mL}$ e agulhas hipodérmicas $25 \times 7 \mathrm{~mm}$ umedecidas com heparina $100 \mathrm{UI}$, 
conforme as preconizaçóes de Ishikawa et al. (2010). Após obtenção das amostras sanguíneas, procedeu-se a dosagem da glicose plasmática pelo método da glicose oxidase e do cloreto plasmático por colorimetria (Doles', Goiás, Brasil). As amostras de plasma para determinação dos íons $\mathrm{Na}^{+}$e $\mathrm{K}^{+}$ foram diluídas 200 vezes em água deionizada, com posterior leitura em fotômetro de chama (B462, Micronal).

Todos os peixes foram sacrificados $(\mathrm{n}=15)$ após os banhos terapêuticos, incluindo os que foram utilizados na coleta sanguínea, sendo as brânquias coletadas e fixadas em formalina $5 \%$ para posterior contagem do número de monogenóides em cada arco branquial com auxílio de microscópio estereoscópico (Tavares-Dias et al. 2001a). A taxa de prevalência e intensidade média de infestação foram calculados segundo Bush et al. (1997).

As diferenças obtidas entre as médias dos diferentes tratamentos foram estabelecidas por análise de variância e as médias comparadas pelo teste de Tukey a 5\% de probabilidade. Todas as análises estatísticas foram realizadas com o software Sigma Stat 3.0.

\section{RESULTADOS E DISCUSSÃO}

No processo de criação intensiva, as alteraçóes na qualidade da água e as práticas de manejo, tais como: manuseio excessivo, transporte e adensamento ainda são consideradas uma importante fonte de estresse para os peixes, podendo inclusive em funçáo da sucessão de estímulos adversos ocasionar alteraçôes no equilíbrio orgânico dos peixes, deixando-os mais susceptíveis a infecçôes parasitárias (Moraes e Martins 2004).
Níveis elevados de glicose, relatados em várias espécies de peixes, é uma das respostas secundárias mais utilizadas para quantificação de estresse nestes organismos (Barton e Iwama 1991; McDonald e Milligan 1997; Wendelaar Bonga 1997). Neste estudo, a glicose plasmática apresentou aumento significativo nos animais tratados com $4 \mathrm{~g} \mathrm{NaCl} \mathrm{L}^{-1}$ por 120 minutos de exposiçáo e nos tratados com 6 e $8 \mathrm{~g} \mathrm{NaCl} \mathrm{L}^{-1}$ por 60 e 120 minutos, em relação aos demais tratamentos (Tabela 1).

Em resposta ao estresse, a mobilizaçáo da glicose ocorre como meio para fornecer energia extra ao animal, para que este possa superar o distúrbio imposto (Barton e Iwama 1991; Wendelaar Bonga 1997). Neste estudo, apesar do aumento nos níveis de glicose acima relatados, esses valores ficaram com uma amplitude de variaçáo de 59 a $80 \mathrm{mg} \mathrm{dL}^{-1}$, estando próximos aos valores normais registrados para tambaquis criados em tanques-rede (Chagas et al. 2003; Gomes et al. 2006). Em situação de estresse agudo, os níveis de glicose de tambaquis podem chegar a 166,9 $\mathrm{mg} \mathrm{dL}^{-1}$ (Tavares-Dias et al. 2001b).

Diminuição na concentração plasmática de íons, como sódio e cloreto, é comumente observada após situações estressantes, como os banhos terapêuticos, em funçâo da elevação nos níveis de catecolaminas induzir o aumento da permeabilidade das brânquias, resultando em alteraçōes nos níveis eletrolíticos sanguíneos em função do gradiente em relaçấo ao meio externo (Eddy 1981). Neste estudo, os tambaquis expostos a $8 \mathrm{~g} \mathrm{NaCl} \mathrm{L}-1$ por 120 minutos apresentaram aumento significativo nos níveis de cloreto plasmático (Tabela 1). Entretanto, os valores observados para

Tabela 1 - Valores médios ( \pm erro padrão) dos parâmetros fisiológicos de juvenis de tambaqui (Colossoma macropomum) tratados com diferentes concentrações de cloreto de sódio em diferentes tempos de exposição.

\begin{tabular}{|c|c|c|c|c|c|}
\hline \multirow{2}{*}{ Tempo de exposição } & \multicolumn{5}{|c|}{ Sal comum $\left(\mathrm{g} \mathrm{L}^{-1}\right)$} \\
\hline & 0 & 2 & 4 & 6 & 8 \\
\hline & \multicolumn{5}{|c|}{ Glicose (mg dL-1) } \\
\hline $30 \mathrm{~min}$ & $59,8 \pm 8,3 a$ & $63,6 \pm 9,9 a$ & $66,4 \pm 11,9 a$ & $64,9 \pm 14,3 a$ & $71,0 \pm 6,3 a$ \\
\hline $60 \min$ & $64,8 \pm 3,9 b$ & $66,6 \pm 1,8 b$ & $67,2 \pm 5,7 b$ & $76,7 \pm 12,5 \mathrm{a}$ & $72,9 \pm 14,5 a$ \\
\hline \multirow[t]{2}{*}{$120 \mathrm{~min}$} & $64,6 \pm 2,6 b$ & $66,4 \pm 6,0 b$ & $76,32 \pm 15,4 a$ & $80,5 \pm 11,7 a$ & $80,4 \pm 12,6 a$ \\
\hline & \multicolumn{5}{|c|}{ Cloreto (mEq L-1) } \\
\hline $30 \mathrm{~min}$ & $127,4 \pm 47,8 a$ & $137,8 \pm 50,5 a$ & $116,1 \pm 12,1 \mathrm{a}$ & $123,5 \pm 10,2 \mathrm{a}$ & $133,4 \pm 20,8 a$ \\
\hline $60 \mathrm{~min}$ & $131,1 \pm 24,4 a$ & $121,4 \pm 10,2 \mathrm{a}$ & $134,2 \pm 28,6 a$ & $135,7 \pm 29,8 a$ & $137,4 \pm 31,9 a$ \\
\hline \multirow[t]{2}{*}{$120 \mathrm{~min}$} & $127,9 \pm 18,9 b$ & $136,5 \pm 18,9 b$ & $153,2 \pm 34,5 b$ & $171,5 \pm 64,6 b$ & $185,9 \pm 37,4 a$ \\
\hline & \multicolumn{5}{|c|}{ Sódio (mEq L-1) } \\
\hline $30 \mathrm{~min}$ & $128,2 \pm 17,8 \mathrm{a}$ & $144,1 \pm 29,2 a$ & $139,8 \pm 19,2 a$ & $137,4 \pm 20,1 a$ & $148,3 \pm 20,8 a$ \\
\hline $60 \min$ & $138,9 \pm 12,9 a$ & $126,9 \pm 14,8 \mathrm{a}$ & $132,9 \pm 14,9 a$ & $155,4 \pm 49,3 a$ & $132,9 \pm 16,3 a$ \\
\hline \multirow[t]{2}{*}{$120 \mathrm{~min}$} & $128,0 \pm 17,4 \mathrm{a}$ & $117,8 \pm 12,4 a$ & $140,8 \pm 33,2 \mathrm{a}$ & $141,7 \pm 9,1 \mathrm{a}$ & $138,3 \pm 8,0 \mathrm{a}$ \\
\hline & \multicolumn{5}{|c|}{ Potássio (mEq L-1) } \\
\hline $30 \mathrm{~min}$ & $5,9 \pm 0,3 a$ & $5,9 \pm 0,8 a$ & $5,8 \pm 0,7 a$ & $5,9 \pm 0,6 a$ & $5,7 \pm 0,6 a$ \\
\hline $60 \mathrm{~min}$ & $6,0 \pm 0,8 \mathrm{a}$ & $5,8 \pm 0,9 a$ & $5,2 \pm 0,9 a$ & $5,1 \pm 0,7 a$ & $4,9 \pm 1,1 \mathrm{a}$ \\
\hline $120 \mathrm{~min}$ & $5,5 \pm 1,5 a$ & $4,9 \pm 0,9 \mathrm{a}$ & $5,3 \pm 1,2 a$ & $5,9 \pm 0,8 a$ & $4,9 \pm 0,8 a$ \\
\hline
\end{tabular}

Médias seguidas da mesma letra, nas linhas, não diferem entre si a $5 \%$ de probabilidade, pelo teste de Tukey. 
o tambaqui (136,5 a $\left.185,9 \mathrm{mEq} \cdot \mathrm{L}^{-1}\right)$ são muito próximos aos valores basais $\left(120,05 \mathrm{mEq} \mathrm{L}^{-1}\right)$ descritos para juvenis de matrinxã (Urbinati et al. 2004).

Com relação aos íons sódio e potássio, não houve diferença significativa para os níveis plasmáticos destes íons nos diferentes tratamentos com sal $\left(0,2,4,6\right.$ e $\left.8 \mathrm{~g} \mathrm{~L}^{-1}\right)$. Em estudos com o híbrido híbrido Morone chrysops x M. saxatilis, transportados com $8 \mathrm{~g} \mathrm{NaCl} \mathrm{L}^{-1}$ de água, não foi observada disfunção osmorregulatória, sugerindo que essa concentração de sal é semelhante a concentração osmótica interna, diminuindo assim o gradiente iônico entre o ambiente externo e interno, sugerindo que o peixe economiza certa quantidade de energia que é utilizada para o balanço iônico em situaçôes de estresse (Weirich et al. 1992).

Com relação às doenças parasitárias, não há disponibilidade de dados sobre os prejuízos acarretados por estas doenças na piscicultura brasileira. Entretanto, sabese que os monogenóides são os que causam as lesões mais severas em peixes cultivados (Martins et al. 2000; Martins et al. 2001; Tavares-Dias et al. 2001a), ocasionando problemas respiratórios graves (Tavares-Dias et al. 2000), sendo considerados um obstáculo à produtividade dos animais em criação intensiva.

Neste estudo, a prevalência de monogenóides nas brânquias dos juvenis de tambaqui no início do período experimental foi de 100\% (Tabela 2). As espécies encontradas foram A. spathulatus, $N$. janauachensis e Mymarothecium boegeri. Estas espécies também foram relatadas para tambaquis criados em sistema de tanques-rede em diferentes densidades de estocagem (Varella et al. 2003; Morais et al. 2009).

Os protocolos de tratamentos de doenças em peixes comumente empregados consistem na realização de banhos terapêuticos, uso de raçôes medicadas e aplicaçáo de vacinas (Thune et al. 1997; Wise e Johnson 1998; Wise et al. 2000;

Tabela 2 - Valores médios ( \pm erro padrão) de monogenóides encontrados nas brânquias de tambaqui (Colossoma macropomum) tratados com diferentes concentrações de cloreto de sódio independente do tempo de exposição.

\begin{tabular}{cccc}
$\begin{array}{c}\text { Cloreto de sódio } \\
\left(\mathrm{g} . \mathrm{L}^{-1}\right)\end{array}$ & $\begin{array}{c}\text { Número total } \\
\text { de parasitas }\end{array}$ & $\begin{array}{c}\text { Prevalência } \\
(\%)\end{array}$ & $\begin{array}{c}\text { Intensidade média } \\
\text { de infestação }\end{array}$ \\
\hline $\begin{array}{c}\text { Antes dos } \\
\text { banhos }\end{array}$ & 525 & 100 & $58,38 \pm 9,65$ \\
0 & 429 & 100 & $47,66 \pm 5,16 \mathrm{a}$ \\
\hline 2 & 347 & 100 & $38,55 \pm 5,91 \mathrm{a}$ \\
4 & 403 & 100 & $44,66 \pm 8,04 \mathrm{a}$ \\
6 & 375 & 100 & $41,66 \pm 8,01 \mathrm{a}$ \\
\hline 8 & 569 & 100 & $63,22 \pm 15,08 \mathrm{a}$ \\
\hline
\end{tabular}

Médias seguidas da mesma letra, nas colunas, não diferem entre si a $5 \%$ de probabilidade, pelo teste de Tukey.
Fouz et al. 2001; Wise e Terhune 2001). O sal comum é um dos banhos mais utilizados na profilaxia de peixes, pois, além de reduzir o estresse (Wurts 1995; Gomes et al. 2003; Urbinati e Carneiro 2004), tem eficácia comprovada na prevenção e no tratamento de doenças (Pavanelli et al. 2008). Neste estudo, houve prevalência de $100 \%$ de helmintos monogenóides nas brânquias dos tambaquis após a exposição aos banhos com sal comum, não sendo observada diferença significativa no número médio de monogenóides entre as diferentes concentraçôes de sal $\left(0,2,4,6\right.$ e $\left.8 \mathrm{~g} \mathrm{~L}^{-1}\right)$ utilizadas nos diferentes tempos de exposição (Tabela 2); portanto, não sendo observada eficácia do emprego do sal no controle de helmintos monogenóides em tambaqui. De forma semelhante ao observado nesse estudo, Vargas et al. (2003) relataram que o tratamento com sal a 3\% durante 10 minutos para tilápia do Nilo (Oreochromis niloticus) não foi eficaz na diminuição da ocorrência e nem na intensidade de ocorrência de helmintos monogenóides.

Para outros grupos de parasitas, como os protozoários Ichthyophthirius multifliiis e Piscinoodinium pillulare foram encontrados efeitos benéficos após o emprego do sal em protocolos de banhos de imersão. Para juvenis de jundiá (Rhamdia quelen), houve redução na taxa de mortalidade e no número do parasita Ichthyophtirius multifiliis após a realização de três banhos terapêuticos com sal na concentração de 10 $\mathrm{g} \mathrm{L}^{-1}$ (Carneiro et al. 2005). Ainda, o emprego do sal no transporte de matrinxấs, na concentração de $6 \mathrm{~g} \mathrm{~L}^{-1}$, foi efetivo no controle de Piscinoodinium sp. (Carneiro et al. 2002).

É importante ressaltar que apesar do sal comum ser um produto barato, prontamente disponível, relativamente seguro para uso, efetivo na redução do estresse osmorregulatório e contra uma ampla variedade de parasitas, dentre eles os helmintos monogenóides (Kabata 1985; Gomes et al. 2003; Urbinati e Carneiro 2004; Pavanelli et al. 2008), neste estudo as concentraçōes avaliadas não foram eficazes na redução de monogenóides em tambaqui, além de promover elevação da glicose plasmática ( $4 \mathrm{~g} \mathrm{NaCl} \mathrm{L}^{-1}$ por 120 minutos, 6 e $8 \mathrm{~g} \mathrm{NaCl}$ $\mathrm{L}^{-1}$ por 60 e 120 minutos) e dos níveis de cloreto plasmático (8 $\mathrm{g} \mathrm{NaCl} \mathrm{L}^{-1}$ por 120 minutos). Entretanto, futuros estudos devem ser realizados avaliando outras espécies de peixes e que abrigam diferentes espécies e gêneros de helmintos, os quais podem apresentar diferentes graus de susceptibilidade.

\section{AGRADECIMENTOS}

Aos Srs. Cristhian Pérez, Andrezza Chagas, Gregório Mota e Edílson Araújo pelo auxílio na condução dos experimentos, análises fisiológicas e parasitológicas. Ao apoio financeiro do projeto TANRE FINEP/FUCAPI e BASA. Ao CNPq pela bolsa de produtividade em pesquisa concedida aos autores L.C. Gomes e J.C.O. Malta. 


\section{BIBLIOGRAFIA CITADA}

Araújo, C.S.O.; Gomes, A.L.; Tavares-Dias, M.; Andrade, S.M.S.; Belém, A.C.; Borges, J.T.; Queiroz, M.A.; Barbosa, M. 2009. Parasitic infections in pirarucu fry, Arapaima gigas Schinz, 1822 (Arapaimatidae) kept in a semi-intensive fish farm in Central Amazon, Brazil. Veterinarski Arhiv, 79: 499-507.

Araújo-Lima, C.; Gomes, L.C. 2005. Tambaqui Colossoma macropomum, p. 175-202. In: Baldisserotto, B.; Gomes, L.C. (Eds.). Espécies nativas para piscicultura no Brasil. v.1. Universidade Federal de Santa Maria, Santa Maria, Rio Grande do Sul.

Barton, B.A.; Iwama, G.K. 1991. Physiological changes in fish from stress in aquaculture with emphasis on the response and effects of corticosteroids. Annual Review of Fish Disease, 1: 3-26.

BRASIL. Ministério da Pesca e Aquicultura. 2010. Boletim estatístico da pesca e aquicultura - Brasil 2008 - 2009. Brasília, DF. 99 pp.

Bush, A.O.; Lafferty, K.D.; Lotz, J.M.; Shostak, A.W. 1997. Parasitology meets ecology on its own terms: Margolis et al. Revisited. Journal of Parasitology, 83: 575-583.

Carneiro, P.C.F.; Martins, M.L.; Urbinati, E.C. 2002. Effect of sodium chloride on physiological responses and the gill parasite, Piscinoodinium sp., in matrinxã, Brycon cephalus, (Teleostei: Characoidei) subjected to transport stress. Journal of Aquaculture in the Tropics, 17: 337-348.

Carneiro, P.C.F.; Schorer, M.; Mikos, J.D. 2005. Tratamentos terapêuticos convencionais no controle do ectoparasita Ichthyophtirius multifliis em jundiá (Rhamdia quelen). Pesquisa Agropecuária Brasileira, 40: 99-102.

Chagas, E.C.; Lourenço, J.N.P.; Gomes, L.C.; Val, A.L. 2003. Desempenho e estado de saúde de tambaquis cultivados em tanques-rede sob diferentes densidades de estocagem, p. 83-93. In: Urbinati, E.C.; Cyrino, J.E.P. (Eds.). XII Simpósio Brasileiro de Aqüicultura. v.2. AQUABIO, Jaboticabal, São Paulo.

Eddy, F.B. 1981. Effects of stress on osmotic and ionic regulation in fish, p. 77-102. In: Pickering A.D. (Ed). Stress and fish. Academic Press.

Fouz, B.; Esteve-Gassent, M.D.; Barreira, R.; Larsen, J.L.; Nielsen, M.E.; Amaro, C. 2001. Field testing of a vaccine against eel diseases caused by Vibrio vulnificus. Diseases of Aquatic Organisms, 45: 183-189.

Gomes, L.C.; Chippari-Gomes, A.R.; Lopes, N.P.; Roubach, R.; Araújo-Lima, C.A.R.M. 2001. Efficacy of benzocaine as an anesthetic in juvenile tambaqui, Colossoma macropomum. Journal of the World Aquaculture Society, 32: 426-431.

Gomes, L.C.; Araújo-Lima, C.A.R.M; Roubach, R.; Urbinati, E.C. 2003. Avaliação dos efeitos da adiçáo de sal e da densidade no transporte de tambaqui. Pesquisa Agropecuária Brasileira, 38 : 283-290.

Gomes, L.C.; Chagas, E.C.; Martins, H.; Roubach, R.; Ono, E.A.; Lourenço, J.N.P. 2006. Cage culture of tambaqui (Colossoma macropomum) in a central Amazon floodplain lake. Aquaculture, 253: 374-384.
Instituto Brasileiro do Meio Ambiente e dos Recursos Naturais Renováveis - IBAMA. 2005. Produçâo brasileira da Aqüicultura Continental, por Estado e espécie, para o ano de 2005: Estatística da Aqüicultura e Pesca no Brasil - Ano 2005. Brasília: SEAP. 101 pp.

Instituto Brasileiro do Meio Ambiente e dos Recursos Naturais Renováveis - IBAMA. 2007. Produção brasileira da Aqüicultura Continental, por Estado e espécie, para o ano de 2007: Estatística da Pesca no Brasil - Ano 2007. Brasília: IBAMA. 113 pp.

Ishikawa, M.M.; Pádua, S.B.; Satake, F.; Pietro, P.S.M.; Hisano, H. 2010. Procedimentos básicos para colheita de sangue em peixes. Embrapa Agropecuária Oeste, Dourados. 8 pp.

Kabata, Z. 1985. Parasites and diseases of fish cultured in the tropics. Taylor \& Francis, London. 318 pp.

Maciel, P.O.; Affonso, E.G.; Boijink, C.L.; Tavares-Dias, M.; Inoue, L.A.K.A. 2011. Myxobolus sp. (Myxozoa) in the circulating blood of Colossoma macropomum (Osteichthyes, Characidae). Revista Brasileira de Parasitologia Veterinária, 21: 80-82.

Malta, J.C.O.; Gomes, A.L.S.; Andrade, S.M.S.; Varella, A.M.B. 2001. Infestações maciças por acantocéfalos, Neoechinorhynnchus buttnerae GOLVAN, 1956, (EOACANTHOCEPHALA, NEOECHINORHYNCHIDAE) em tambaquis jovens, Colossoma macropomum (CUVIER, 1818) cultivados na Amazônia Central. Acta Amazônica, 31: 133-143.

Martins, M.L.; Moraes, F.R.; Fujimoto, R.Y.; Onaka, E.M.; Nomura, D.T.; Silva, C.A.H.; Schalch, S.H.C. 2000. Parasitic infections in cultivated freshwater fishes a survey of diagnosticated cases from 1993 to 1998. Revista Brasileira de Parasitologia Veterinária, 9: $23-28$

Martins, M.L.; Moraes, J.R.E.; Andrade, P.M.; Schalch, S.H.C.; Moraes, F.R. 2001. Piscinoodinium pillulare (SCHAPERCLAUS, 1954) Lom, 1981 (DINOFLAGELLIDA) infection in cultivated freshwater fish from the northeast region of São Paulo State, Brasil. Parasitological and pathological aspects. Brazilian Journal of Biology, 61: 639-644.

McDonald, D.G.; Milligan, C.L. 1997. Ionic, osmotic and acidbase regulation in stress, p. 119-144. In: Iwama, G.K.; Pickering, A.D.; Sumpter, J.P.; Schreck, C.B. (Eds.). Fish stress and health in aquaculture. Cambridge University Press, Cambridge.

Melo, L.A.S.; Izel, A.C.U.; Rodrigues, F.M. 2001. Criação de tambaqui (Colossoma macropomum) em viveiros de argila/barragens no Estado do Amazonas. Embrapa Amazônia Ocidental, Manaus, AM. 25 pp.

Moraes, F.R.; Martins, M.L. 2004. Condições predisponentes e principais de enfermidades de teleósteos em piscicultura intensiva, p. 343-386. In: Cyrino, J.E.P.; Urbinati, E.C.; Fracalossi, D.M.; Castagnolli, N. (Eds.). Tópicos especiais em piscicultura de água doce tropical intensiva. Sociedade Brasileira de Aqüicultura e Biologia Aquática, Jaboticabal, São Paulo.

Morais, A.M.; Correa, M.A.V.; Varella, A.M.B.; Malta, J.C.O. 2009. A fauna de parasitas de juvenis de tambaqui Colossoma macropomum (Cuvier, 1818) (Characidae: Serrasalmidae) criados em tanques-rede em um lago de várzea da Amazônia central. Biologia Geral e Experimental, 9: 13-23. 
Pavanelli, G.C.; Eiras, J.C.; Takemoto, R.M. 2008. Doenças de peixes: profilaxia e tratamento. EDUEM, Maringá, PR. 311 pp.

Saint-Paul, U. 1986. Potential for aquaculture of South American fresh water fishes: a review. Aquaculture, 54: 205-240.

Silva, C.M.A. 2001. Bactérias gram-negativas isoladas do tambaqui, Colossoma macropomum (Cuvier, 1818) criado em cativeiro, Amazonas-Brasil. Dissertação de Mestrado, Instituto Nacional de Pesquisas da Amazônia/Fundação Universidade do Amazonas, Manaus, Amazonas. 66 pp.

Tavares-Dias, M; Schalch, S.H.C.; Martins, M.L.; Moraes, F.R. 2000. Característica hematológicas de Oreochromis niloticus (Osteichthyes: Cichlidae) cultivadas intensivamente em "pesquepague" do município de Franca, São Paulo, Brasil. Ars Veterinária, 16: 76-82.

Tavares-Dias, M.; Moraes, F.R.; Martins, M.L.; Kronka, S.N. 2001 a. Fauna parasitária de peixes oriundos de pesque-pagues do município de Franca, São Paulo, Brasil. II. Metazoários. Revista Brasileira de Zoologia, 18: 81-95.

Tavares-Dias, M.; Sandrim, E.F.S.; Moraes, F.R.; Falanghe, P.C. 2001b. Physiological responses of "Tambaqui" Colossoma macropomum (CHARACIDAE) to acute stress. Boletim do Instituto de Pesca, 27: 43-48.

Tavechio, H.L.G.; Guidelli, G.; Portz, L. 2009. Alternativas para a prevençáo e o controle de patógenos em piscicultura. Boletim do Instituto de Pesca, 35: 335-341.

Thatcher, V.E.; Brites Neto, J. 1994. Diagnóstico, prevenção e tratamento das enfermidades de peixes neotropicais de água doce. Revista Brasileira de Medicina Veterinária, 16: 111-128.

Thatcher, V.E. 2006. Amazon fish parasites. Pensoft Publishers, Moscow, Russian. 508 pp.

Thune, R.L.; Collins, L.A.; Pena, M.P.A. 1997. A Comparison of immersion, immersion/oral combination and injection methods for the vaccination of channel catfish Ictalurus punctatus against Edwardsiella ictaluri. Journal of the Word Aquaculture Society, 28: 193-201.

Urbinati, E.C.; Abreu, J.S.; Camargo, A.C.S.; Landines, M.A. 2004. Loading and transport stress in juvenile matrinxã (Brycon cephalus) at various densities. Aquaculture, 229: 389-400.

Urbinati, E.C.; Carneiro, P.C.F. 2004. Práticas de manejo e estresse dos peixes em piscicultura, p. 343-386. In: Cyrino, J.E.P.; Urbinati, E.C.; Fracalossi, D.M.; Castagnolli, N. (Eds.). Tópicos especiais em piscicultura de água doce tropical intensiva. Sociedade Brasileira de Aqüicultura e Biologia Aquática, Jaboticabal, São Paulo.
Val, A.L.; Silva, M.N.P.; Almeida-Val, V.M.F. 1998. Hypoxia adaptation in fish of the Amazon: a never-ending task. South African Journal of Zoology, 33: 107-114.

Varella, A.M.B.; Peiro, S.N.; Malta, J.C.O.; Lourenço, J.N.P. 2003. Monitoramento da parasitofauna de Colossoma macropomum (Cuvier, 1818) (Osteichthyes: Characidae) cultivado em tanquesrede em um lago de varzea na Amazônia, p. 95-106. In: Urbinati, E.C.; Cyrino, J.E.P. (Eds.). XII Simpósio Brasileiro de Aqüicultura. AQUABIO, Jaboticabal, São Paulo.

Vargas, L.; Povh, J.A.; Ribeiro, R.P.; Moreira, H.L.M.; Rocha Loures, B.T.R.; Maroneze, M.S. 2003. Efeito do tratamento com cloreto de sódio e formalina na ocorrência de ectoparasitas em alevinos de tilápia do Nilo (Oreochromis niloticus) revertidos sexualmente. Arquivo de Ciências Veterinárias e Zoologia, 6: 39-48.

Weirich, C.R.; Tomasso, J.R.; Smith, T.I.J. 1992. Confinement and transport-induced stress in white bass Morone chrysops x striped bass M. saxatilis hybrids: Effect of calcium and salinity. Journal of the World Aquaculture Society, 2: 49-57.

Wendelaar Bonga, S.E. 1997. The stress response in fish. Physiological Reviews, 77: 591-625.

Wise, D.J.; Johnson, M.R. 1998. Effect of feeding frequency and romet-medicat feed on survial, antibody response, and weight gain of fingerling channel catfish Ictalurus punctatus aftrer natural exposure to Edwardsiella ictaluri (re-33). Journal of the World Aquaculture Society, 29: 169-175.

Wise, D.J.; Terhune, J.S. 2001. The relationship between vaccine dose and efficacy in channel catfish Ictalurus punctatus vaccinated as fry with a live attenuated strain of Edwardsiella ictaluri (re-33). Journal of the World Aquaculture Society, 32: 177-183.

Wise, D.J.; Klesius, P.H.; Shoemaker, C.A.; Wolters, W.R. 2000. Vaccination of mixed an full-sib families of channel catfish Ictalurus punctatus against enteric septicemia of catfish with a live attenuated Edwardsiella ictaluri isolate (re-33). Journal of the World Aquaculture Society, 31: 206-212.

Wurts, W.A. 1995. Using salt to reduce handling stress in channel catfish. World Aquaculture, 26: 80-81.

Recebido em: 08/04/2011

Aceito em: 04/07/2011 